\title{
Diazonium electrografting vs. physical adsorption of Azure A at carbon nanotubes for mediated glucose oxidation with FAD-GDH
}

Andrew J. Gross,[a]* Shunya Tanaka,[a,b] Clara Colomies,[a] Fabien Giroud,[a] Yuta

Nishina,[c] Serge Cosnier,[a] Seiya Tsujimura,[b] and Michael Holzinger[a]*

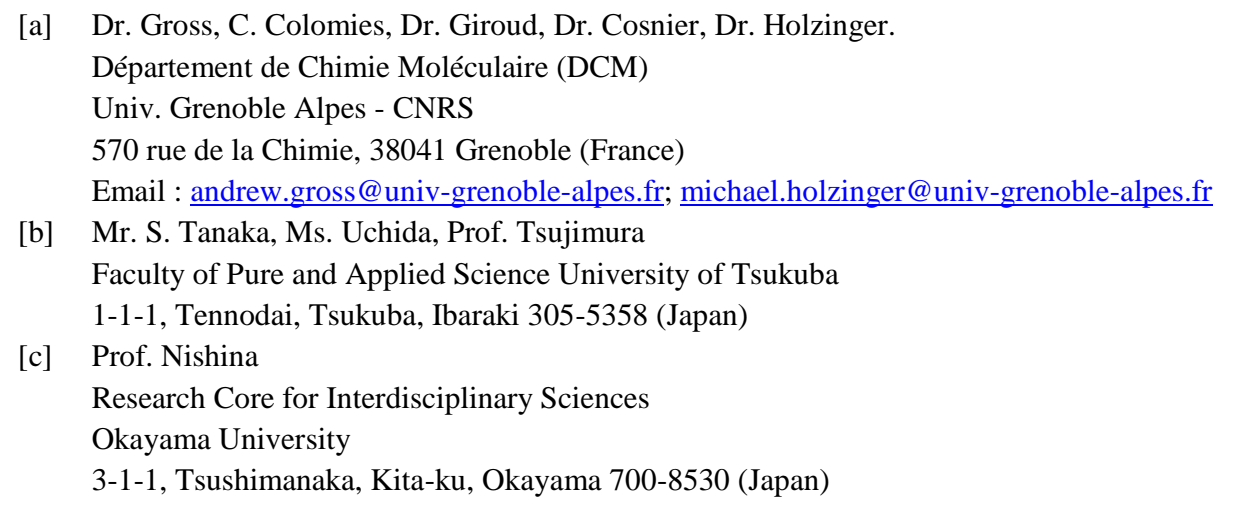

Abstract : The electrochemical reduction of aryldiazonium salts is a versatile and direct route to obtain robust covalently-modified electrodes. We report here a comparative study of Azure-A modified carbon nanotube electrodes prepared by diazonium electrografting and by physical adsorption for bioelectrocatalytic glucose oxidation with fungal FAD-glucose dehydrogenase from Aspergillus sp. The electrografted and adsorbed electrodes exhibited different reversible electroactivity consistent with polymer-type and monomer-type phenothiazine surface assemblies, respectively. The electrografted Azure A electrodes exhibited superior mediated bioelectrocatalysis compared to the adsorbed Azure A electrodes. A more than 10-fold higher catalytic current up to $2 \mathrm{~mA} \mathrm{~cm}^{-2}$ at $0.2 \mathrm{~V} v$. $\mathrm{Ag} / \mathrm{AgCl}$ together with a similarly low onset potential of $-0.05 \mathrm{~V} v s$. $\mathrm{Ag} / \mathrm{AgCl}$ was observed at the electrografted electrodes. Faster estimated electron transfer kinetics and a $+200 \mathrm{mV}$ potential shift for the polymer-type redox couple vs. the adsorbed monomer-type couple underlines the favourable driving force for mediated electron transfer with the buried FAD active site for the diazonium-derived bioelectrode.

Keywords: Phenothiazine redox mediator $\bullet$ FAD dependant glucose dehydrogenase $\bullet$ biofuel cell anode $\bullet$ enzymatic bioelectrode $\bullet$ bioelectrocatalysis 


\section{Introduction}

The development of bioelectrodes based on carbon nanotubes (CNTs) with immobilised oxidoreductases is of great interest for the development of enzymatic biofuel cells $(\text { EBFCs })^{[1,2]}$. EBFCs are eco-friendly power sources that exploit electrically wired enzymes to generate electricity from chemical energy via bioelectrocatalytic reactions. The most typical and arguably most promising EBFCs rely on glucose oxidation at the bioanode and oxygen reduction at the biocathode. Recent developments in EBFC design demonstrate the practical possibility for powering electronic devices such as an implanted temperature sensor or portable ovulation test ${ }^{[3,4]}$. Despite great promise, there are still many challenges to overcome, in particular relating to the development of catalytic bioelectrodes with high surface area and stability, efficient electron transfer, low overpotential, fast mass transport, and high current density.

Glucose oxidase (GOx) has been the gold standard catalyst for the bioelectrocatalytic oxidation of glucose in biofuel cells due to its high thermal stability and superior selectivity for glucose $e^{[4,5]}$. Fungal-based flavin adenine dinucleotide (FAD)-dependent glucose dehydrogenase (FAD-GDH) has emerged as a powerful alternative for glucose-oxidising bioanodes due to high turnover rates and substrate selectivity, good stability, a low redox potential, and oxygen insensitivity ${ }^{[5-7]}$. Unlike GOx, FAD-GDH does not use molecular oxygen as an electron acceptor. The consumption of oxygen (oxidant) at the anode, as well as the resulting enzymatic production of hydrogen peroxide, can thus be avoided. Hydrogen peroxide is a known inhibitor for multicopper oxidase (MCO)-based biocathodes commonly employed in biofuel cells for bioelectrocatalytic oxygen reduction to water ${ }^{[6,8,9]}$. 
As is the case with GOx, the FAD cofactor of fungal FAD-GDH is surrounded by an insulating protein shell and typically requires a redox mediator for electron transfer with the electrode. Metal complexes and quinones with suitable potentials and affinities for the FAD active site have been particularly exploited as redox mediators for FAD-GDH. For example, osmium- $^{[7,10]}$ and ruthenium- ${ }^{[11,12]}$ based complexes, and derivatives of 1,2 and 1,4 naphthoquinone $\mathrm{e}^{[5,6,13]}, 1,10$-Phenanthroline-5,6-dione $\mathrm{e}^{[6]}$ and 1,6 and 1,8 pyrenedione ${ }^{[14]}$ have been developed. Phenothiazines were recently proposed as either diffusional or immobilised mediators with attractive formal potential and electron transfer kinetics for fungal FAD$\mathrm{GDH}^{[15,16]}$. Schumann et al. showed the possibility of using phenothiazine redox polymers for wiring FAD-dependent enzymes for glucose oxidation, but this was for cellobiose dehydrogenase or glucose oxidase, not for FAD-GDH ${ }^{[17]}$. Redox mediators for FAD-GDH have been immobilised at the electrode surface to infer stability either by physical adsorption via electrostatic and/or pi-pi interactions ${ }^{[6,11,14]}$, or by covalent tethering to the backbone of a polymer $^{[5,7,10,12,13,16]}$. We recently reported an alternative strategy based on direct anodic electrografting of poly(methylene green) as a polymeric mediator for fungal FAD-GDH at glassy carbon (GC) and MgO-templated carbon (MgOC) electrodes ${ }^{[18]}$.

In this study, we explore a mediator immobilisation strategy based on the electrochemical reduction of the in-situ generated diazonium salt derivative of Azure A to yield redox mediator assemblies at carbon nanotube electrodes for application in bioelectrocatalysis. Aryldiazonium salt electrografting is a convenient radical-based approach to obtain covalently-functionalised carbon electrodes with high stability and usually multilayer film structures with a high level of control ${ }^{[19,20]}$. It is well known that the electrochemical reduction of aryldiazonium salt ions to aryl radicals leads to aryl-carbon surface covalent bonding, for example, when electrografting in aqueous acidic media or non-nucleophilic aprotic 
solvents $^{[21]}$. Polymeric layers with thicknesses typically between 1 and $20 \mathrm{~nm}$ are formed ${ }^{[22]}$. A wide variety of carbon substrates have been modified by diazonium grafting including GC, graphene oxide, pyrolysed photoresist film (PPF) and carbon nanotubes ${ }^{[20,21,23-26]}$. Mechanistic aspects of the reaction of the aryl radicals at carbon nanotubes have been studied $^{[26,27]}$. Azure A is a convenient choice for diazonium grafting due to its single pendant arylamine group. Notably, diazonium-derived Azure A electrodes with a stable and reversible response have been reported at screen-printed carbon for the electrocatalytic oxidation of $\mathrm{NADH}^{[28]}$. Diazonium-derived Azure A electrodes have also been investigated at graphene oxide and gold electrodes ${ }^{[25]}$. Few examples of diazonium-derived electrodes have been reported for the preparation of bioelectrodes in EBFC design ${ }^{[29,30]}$. Minteer and coworkers also reported the use of anodically polymerised Azure A and other phenothiazines for NAD oxidation and extended this for glucose oxidation at a bioanode with $\mathrm{NAD}^{+}$dependent $\mathrm{GDH}^{[31]}$. To the best of knowledge, aryldiazonium salt surface modification has not been reported for the construction of a catalytic bioelectrode with electrically-wired FAD-GDH. In addition to exploring the use of diazonium electrografting, we also report here a comparison with a more classical physical adsorption method, to reveal new insight into the use of different functionalisation strategies for mediated bioelectrocatalysis.

\section{Experimental Section}

\subsection{Materials and chemicals}

Monopotassium phosphate monohydrate $\left(\mathrm{KH}_{2} \mathrm{PO}_{4}, \geq 98 \%\right)$, dipotassium hydrogen phosphate heptahydrate $\left(\mathrm{K}_{2} \mathrm{HPO}_{4}, 98-102 \%\right)$, Azure A chloride certified dye (88\%), 1-Methyl-2pyrrolidone (NMP, $\geq 99 \%)$, sodium nitrite $\left(\mathrm{NaNO}_{2}, \geq 97 \%\right)$, D-(+)-glucose ( $\left.\geq 99.5 \%\right)$ were purchased from Sigma-Aldrich and used as received. Hydrochloric acid ( $\mathrm{HCl}, 37 \%)$ was purchased from Fisher Scientific. Flavin adenine dinucleotide-dependent glucose 
dehydrogenase (FAD-GDH, $1150 \mathrm{U} \mathrm{mg}^{-1}$ solid) from Aspergillus sp. was purchased from Sekisui Diagnostics (UK) and used without further purification. The enzyme was stored at $-20{ }^{\circ} \mathrm{C}$ when not in use. Glucose stock solutions were left to mutarotate overnight to $\beta$-Dglucose prior to use. Distilled water was purified to a minimum resistivity of $15 \mathrm{M} \Omega \mathrm{cm}^{-1}$ using a Millipore Ultrapure system. Commercial grade multiwalled carbon nanotubes (MWCNTs, $\varnothing=9.5 \mathrm{~nm}, 1.5 \mu \mathrm{m}$ length, $\geq 95 \%$ purity) were obtained from Nanocyl and used as received without purification. High purity oxygen and argon were obtained from Messer.

\subsection{Electrochemistry}

Electrochemical measurements were performed using an Eco Chemie Autolab PGSTAT 11 potentiostat running Nova 2.1 software or a Biologic VMP3 Multi Potentiostat with EC-lab software. Experiments were performed at room temperature unless otherwise stated. A conventional three-electrode cell setup was used comprising a glassy carbon (GC) or modified GC working electrode $(\varnothing=3 \mathrm{~mm})$ polished or modified, a silver-silver chloride reference electrode $(\mathrm{Ag} / \mathrm{AgCl}$ with saturated $\mathrm{KCl})$, and a Pt wire counter-electrode. $\mathrm{GC}$ electrodes were polished using a Presi polishing cloth with $1 \mu \mathrm{m}$ alumina or diamond slurry then sonicated for $5 \mathrm{~min}$ in distilled water prior to use. Electrochemical characterisation experiments were performed in $0.1 \mathrm{~mol}^{-1}$ phosphate buffer ( $\left.\mathrm{pH} 7\right)$ in the absence of oxygen after purging with argon. Amperometric data was recorded at $E_{\mathrm{p}}=0.2 \mathrm{~V}$ vs. $\mathrm{Ag} / \mathrm{AgCl}$ (sat. $\mathrm{KCl}$ ). For bioelectrocatalytic stability experiments, the bioelectrodes were stored in fresh buffer solution between each amperometric experiment. Surface concentrations were estimated from the anodic peak of $\mathrm{CVs}$ recorded at $10 \mathrm{mV} \mathrm{s}^{-1}$ after fitting a linear baseline using Origin pro 9 peak fitting software. The electron transfer rate constants were determined at Azure-A modified GC electrodes from $\mathrm{CVs}$ recorded up to $60 \mathrm{~V} \mathrm{~s}^{-1}$ in phosphate buffer $(\mathrm{pH} 7)$ according to the Laviron method ${ }^{[38,42]}$ (see Supporting Information). Current density and 
surface concentration data obtained based on the geometric surface area of the working electrode $\left(0.071 \mathrm{~cm}^{2}\right)$.

\subsection{Scanning Electron Microscopy}

High resolution imaging of the carbon nanotube electrode morphology was performed using a FEI/Quanta FEG 250 scanning electron microscope (SEM, Hillsboro, OR, USA) with an accelerating voltage of $3 \mathrm{kV}$.

\subsection{Preparation of multiwalled carbon nanotube electrodes}

First, $2.5 \mathrm{mg}$ of CNTs were added to $1 \mathrm{~mL}$ of NMP in a sealed glass vial and the resulting suspension sonicated for 30-60 min to achieve a homogeneous dispersion. $20 \mu \mathrm{L}$ of the CNT dispersion was then drop casted onto the GC electrode to obtain a densely and homogeneously coated CNT layer (Figure S8). The modified electrode was subsequently dried under vacuum to obtain the GC-CNT electrode, then rinsed with distilled water prior to use We have previously reported this type of CNT-modified electrode with attractive properties for electrochemical and bioelectrocatalytic studies at GC and carbon cloth substrates $^{[11,14,29]}$. A CNT film thickness of $6 \mu \mathrm{m}$ was determined at carbon $\operatorname{cloth}^{[29]}$.

2.5. Preparation of Azure A modified electrodes by in-situ diazonium salt electrografting (GC-CNT-AA Diaz and GC-AA $\left.A_{\text {Diaz }}\right)$.

A $10 \mathrm{mmol}^{-1}$ Azure A solution was first prepared in $0.25 \mathrm{M} \mathrm{HCl} .3 \mathrm{~mL}$ of this solution was subsequently added into a pear-shaped electrochemical glass cell and cooled with an ice bath to between $2^{\circ} \mathrm{C}$ and $5^{\circ} \mathrm{C} .3 \mathrm{~mL}$ of a freshly prepared $20 \mathrm{mmol}^{-1} \mathrm{NaNO}_{2}$ solution was subsequently added drop-wise and the reaction stirred for 10 minutes in the dark to generate the diazonium salt in-situ. Upon immediate insertion of the working electrode into the solution, the electrografting reaction was initiated by cyclic voltammetry. The optimised 
standard procedure involved recording 10 cycles between $0.5 \mathrm{~V}$ to $-0.3 \mathrm{~V} v$ s. $\mathrm{Ag} / \mathrm{AgCl}$ (sat. $\mathrm{KCl})$ at $100 \mathrm{mV} \mathrm{s}^{-1}$. The electrode was subsequently rinsed with distilled water then preconditioned by recording 15 scans at $100 \mathrm{mV} \mathrm{s}^{-1}$ in phosphate buffer ( $\mathrm{pH} 7$ ) prior to use. For preparation of GC electrodes modified with Azure A (without CNTs), the same electrografting procedure as above was applied except that electrografting was performed to a switching potential of $-0.5 \mathrm{~V} v s . \mathrm{Ag} / \mathrm{AgCl}$ (sat. $\mathrm{KCl}$ ) at $100 \mathrm{mV} \mathrm{s}^{-1}$.

2.6. Preparation of Azure A modified electrodes by physical adsorption (GC-CNT-AA$A_{A d s}$ and $\left.G C-A A_{A d s}\right)$

For the standard procedure, GC or GC-CNT electrodes were incubated in a $0.25 \mathrm{M} \mathrm{HCl}$ solution containing $5 \mathrm{mmol}^{-1}$ Azure A and left overnight. The electrode was subsequently rinsed with distilled water then pre-conditioned by recording 15 scans at $100 \mathrm{mV} \mathrm{s}^{-1}$ in phosphate buffer ( $\mathrm{pH} 7)$ prior to use.

\subsection{Preparation of FAD-GDH modified bioelectrodes}

An enzyme stock solution was first prepared at $2.5 \mathrm{mg} \mathrm{mL}^{-1}$ in $0.1 \mathrm{~mol}^{-1}$ phosphate buffer $(\mathrm{pH}$ 7) and stored at $-18^{\circ} \mathrm{C} .40 \mu \mathrm{L}$ of the stock solution was carefully thawed to room temperature then incubated on the electrode overnight in the fridge at $4{ }^{\circ} \mathrm{C}$. Prior to catalysis experiments, the electrode was rinsed gently with phosphate buffer $(\mathrm{pH} 7)$ to remove weakly adsorbed FAD-GDH.

3. Results and discussion 
The aryldiazonium salt electrografting and physical adsorption strategies for covalent and non-covalent electrode modification with Azure A, respectively, and the subsequent preparation of FAD-GDH bioelectrodes for glucose oxidation, are illustrated in Scheme 1.

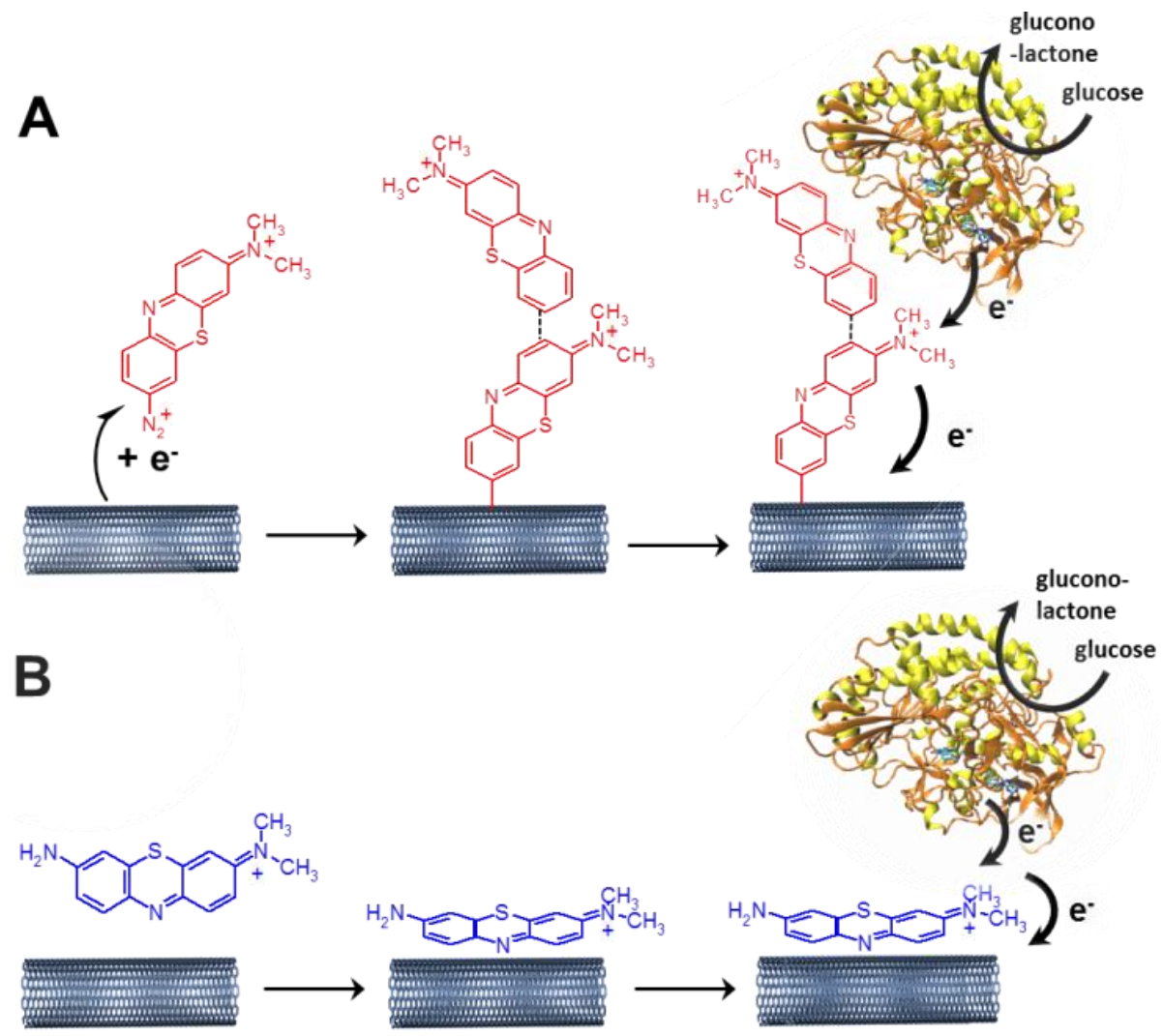

Scheme 1. The preparation strategy and hypothetical structures for (A) diazonium-derived "polymer-type" and (B) physically adsorbed "monomer-type" Azure A bioelectrodes with immobilised fungal FAD-GDH for mediated glucose oxidation.

Diazonium-modified electrodes were obtained via the electrografting of in-situ generated Azure A diazonium salt with an excess of sodium nitrite in $0.25 \mathrm{molL}^{-1} \mathrm{HCl}$ (see Experimental Section). Figure 1A shows a typical example of electrografting performed at multiwalled carbon nanotube-modified glassy carbon (GC-CNT) electrodes. The standard electrografting protocol involved recording 10 cyclic voltammograms (CVs) in the diazonium salt grafting solution from $0.5 \mathrm{~V}$ to $-0.3 \mathrm{~V}$ vs. $\mathrm{Ag} / \mathrm{AgCl}$ at $100 \mathrm{mV} \mathrm{s}^{-1}$. In addition to grafting at low temperature and in the dark, care was taken to ensure electrografting was performed immediately after immersion of the electrode in the grafting solution to minimise any 
unwanted grafting due to the spontaneous diazonium reaction or side reactions involving diazonium or aryl cations ${ }^{[22,27,32]}$. On the first cycle, a broad cathodic peak is observed at $E_{\mathrm{pc}} \approx$ $-0.25 \mathrm{~V} v s . \mathrm{Ag} / \mathrm{AgCl}$, attributed to the irreversible one-electron reduction of the diazonium cation leading to aryl radical generation and subsequent surface modification ${ }^{[19]}$. At positive potentials, two well-defined redox couples with half-wave potentials of $E_{1 / 2}=0.15 \mathrm{~V}$ and 0.29 $\mathrm{V}$ vs. $\mathrm{Ag} / \mathrm{AgCl}$ are observed that increase in magnitude with increasing cycle number, consistent with the growth of redox-active surface assemblies. This modified electrode type is referred to as GC-CNT-AA $A_{\text {Diaz }}$. We note that the current spike observed at the starting potential is a non-faradaic current. A control experiment performed under the same conditions but in the absence of sodium nitrite (and therefore no formed diazonium salt) was also performed (Figure 1B). The first CV cycle shows a different irreversible reduction wave at negative potential that is attributed to non-reversible adsorption of the monomer at the pristine (polymer-free) CNT surface ${ }^{[33]}$. As expected, the control experiment only showed a single reversible redox couple at $E_{1 / 2}=0.15 \mathrm{~V} v s . \mathrm{Ag} / \mathrm{AgCl}$ corresponding to the phenothiazine ring of the Azure A monomer. The increasing current density with cycle number reveals that potential cycling and/or time increases the amount of adsorbed redox-active monomer. For the subsequent comparative study of electrografting $v s$. physical adsorption, we adopted a more classical adsorption modification method based on simple immersion of the electrode in Azure A solution overnight, without potential cycling (see Experimental Section). The adsorbed Azure A electrode prepared by immersion overnight is referred to as GC-CNT$\mathrm{AA}_{\text {Ads }}$. 

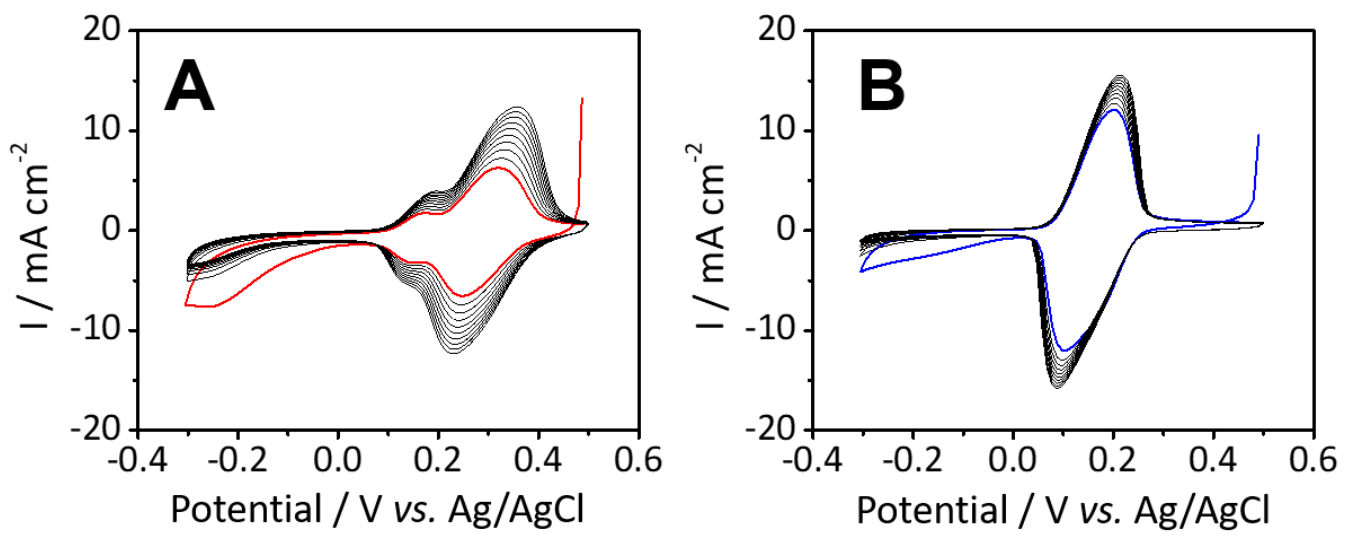

Figure 1. CVs recorded in Azure A $\left(5 \mathrm{mmol}^{-1}\right)$ in $0.25 \mathrm{molL}^{-1} \mathrm{HCl}$ at GC-CNT electrodes of (A) covalent electrografting after in-situ diazonium generation using $\mathrm{NaNO}_{2}\left(10 \mathrm{mmol}^{-1}\right)$, and (B) control performed without $\mathrm{NaNO}_{2}$ addition. Cycle $1(-,-)$ and cycle 2-10 (-). Scan rate $=$ $100 \mathrm{mV} \mathrm{s}^{-1}$.

The GC-CNT-AA Diaz $_{\text {and }}$ GC-CNT-AA Ads modified electrodes were subsequently characterised in $0.1 \mathrm{molL}^{-1}$ phosphate buffer ( $\mathrm{pH}$ 7) solution by cyclic voltammetry. At GCCNT-AA $A_{\text {Diaz }}$, a small redox couple at a low potential of $E_{1 / 2}=-0.27 \mathrm{~V} v s . \mathrm{Ag} / \mathrm{AgCl}$ and a larger redox couple at $E_{1 / 2}=-0.02 \mathrm{~V} v s . \mathrm{Ag} / \mathrm{AgCl}$ are observed (Figure 2A) due to the redox activity of the phenothiazine. The redox couple at lower potential is attributed to a monomertype Azure A assembly and the second redox couple at higher potential is attributed to polymer-type Azure A, based on several studies on polyazine films including electropolymerised Azure A electrodes ${ }^{[34-36]}$. From the difference in half-wave potential for the redox couples observed at $c a . \mathrm{pH} 0.6$ (Figure 1A) and $\mathrm{pH} 7$ (Figure 2B), and assuming ideal Nernstian behaviour, the phenothiazine electroactivity of the GC-CNT-AA redox couples is more consistent with a two-proton two-electron transfer $(-59 \mathrm{mV} / \mathrm{pH})$ rather than a one-proton two-electron transfer $(-30 \mathrm{mV} / \mathrm{pH})$. This is in contrast to the suggested one-proton two-electron phenothiazine electroactivity considered previously for Azure A modified screen-printed carbon ${ }^{[28]}$. The peak-to-peak separations of $\Delta E_{\mathrm{p}} \leq 50 \mathrm{mV}$ at low scan rate (1 to $40 \mathrm{mV} \mathrm{s}^{-1}$ ) and peak symmetry indicate relatively fast apparent electron transfer kinetics for 
the redox couples at GC-CNT electrodes. The observed linear dependence of anodic and cathodic peaks with scan rate provides further evidence that the Azure A is surface-bound (Figure S1). At GC-CNT-AA Ads, a single well-defined redox couple was observed at a low potential of $E_{1 / 2}=-0.25 \mathrm{~V}$ vs. $\mathrm{Ag} / \mathrm{AgCl}$, corresponding to the activity of the adsorbed monomer-type analogue (Figure 2B). The half-wave potential is almost identical to that observed for the low potential redox couple at the electrografted electrode, GC-CNT-AA $A_{\text {Diaz }}$ (Figure 2A). This result signifies the presence of a small amount of adsorbed and/or monomer-type electroactive Azure A at the diazonium-modified electrode surface. Both adsorbed and electrografted Azure A groups showed good stability to repeat cycling (50 cycles) in phosphate buffer ( $\mathrm{pH} 7$ ), as shown in Figure S2. At GC-CNT-AA Diaz, $92 \%$ of the initial activity remained for the polymer-type groups compared to $88 \%$ for the monomer-type groups at GC-CNT-AA $A_{\text {Ads }}$, highlighting similar stability and a possible slight improvement for the diazonium-modified Azure A electrode.
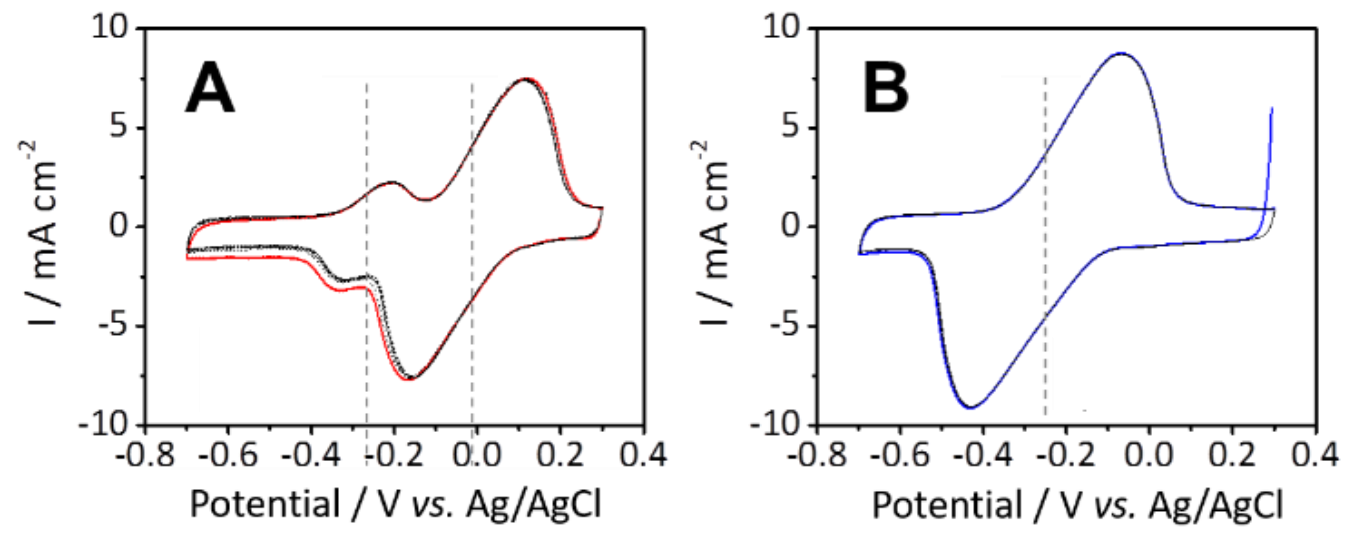

Figure 2. CVs recorded in $0.1 \mathrm{molL}^{-1}$ phosphate buffer $(\mathrm{pH} 7)$ at (A) GC-CNT-AA $A_{\text {Diaz, }}$, prepared by covalent electrografting, and (b) GC-CNT-AA Ads electrodes, prepared by physical adsorption, according to standard protocols. Cycle $1(-,-)$ and cycle 2-10 (-). Scan rate $=100 \mathrm{mV} \mathrm{s}^{-1}$.

The surface concentrations of electroactive Azure A groups at CNT electrodes prepared by electrografting and physical adsorption were subsequently estimated according to Equation 1 
where $\Gamma$ is the surface concentration $\left(\mathrm{mol} \mathrm{cm}^{-2}\right), Q$ is the charge associated with the observed redox process $(\mathrm{C}), n$ is the number of electrons in the redox process (2), $\mathrm{F}$ is the Faraday constant (96485 $\mathrm{C} \mathrm{mol}^{-1}$ ), and A is the geometric area of the working electrode. The value of $Q$ was obtained by dividing the voltammetric peak area value by the scan rate used $\left(0.1 \mathrm{~V} \mathrm{~s}^{-1}\right)$.

$$
\Gamma=\frac{Q}{n F A}
$$

At GC-CNT-AA ${ }_{\mathrm{Ads}}$, an electroactive surface concentration of $\Gamma_{\text {monomer }}=1.0 \times 10^{-7} \mathrm{~mol} \mathrm{~cm}^{-2}$ was observed. This value reflects the total surface concentration of electroactive Azure A. At GC-CNT-AA $_{\text {Diaz }}$, an electroactive surface concentration of $\Gamma_{\text {monomer }}=1.2 \times 10^{-8} \mathrm{~mol} \mathrm{~cm}^{-2}$ and $\Gamma_{\text {polymer }}=7.2 \times 10^{-8} \mathrm{~mol} \mathrm{~cm} \mathrm{~cm}^{-2}$ was observed. The total surface concentration of electroactive groups for the electrografted electrode is therefore $8.4 \times 10^{-8}$ mol cm$~^{-2}$ with $86 \%$ of the redox activity corresponding to the polymeric electrografted groups and a smaller fraction due to adsorbed and/or monomer-type Azure A. The determined surface concentrations are significantly greater than the estimated theoretical value for a densely packed Azure A monolayer in a planar conformation oriented perpendicular to an electrode surface of $\geq 7.6 \times$ $10^{-10} \mathrm{~mol} \mathrm{~cm}{ }^{-2[25]}$. The surface concentrations for the modified electrodes reported here are also significantly greater than previously observed at multiwalled carbon nanotube electrodes $\left(9.2 \times 10^{-10} \mathrm{~mol} \mathrm{~cm}^{-2}\right)^{[37]}$. This is due, at least in part, to the large accessible surface area and favourable porosity of the carbon nanotube network at the electrode surface.

We next considered the electron transfer kinetics of polymer-type (electrografted) vs. monomer-type (adsorbed) Azure A assemblies, according to the well-established Laviron model $^{[38]}$. For these experiments, Azure A assemblies were electrografted and adsorbed at pristine glassy carbon electrodes rather than at CNT electrodes to avoid complexity associated with CNT electrodes being porous and non-planar. The protocol for modification at GC 
electrodes is reported in the Experimental Section. A typical example of the electrografting CVs obtained at GC is presented in Figure S3. The kinetics of the Azure A groups at GC were obtained by recording $\mathrm{CVs}$ at scan rates up to $60 \mathrm{~V} \mathrm{~s}^{-1}$ and extracting the apparent electron transfer rate $\left(k_{\mathrm{s}}\right)$ from the scan rate $(\log ) v s$. peak potential change plots (Figure S4 and Equations S1-S2). The average electron transfer rate constant for the polymer-type electrografted Azure A was $k_{\mathrm{s}}=44.6 \pm 0.8 \mathrm{~s}^{-1}$ compared to a 1.5 fold slower $k_{\mathrm{s}}=30.3 \pm 6.5 \mathrm{~s}^{-1}$ for monomer-type adsorbed Azure A. The rate constant for the electrografted Azure A groups in this work is similar to the $k_{\mathrm{s}} \sim 50 \mathrm{~s}^{-1}$ obtained by experimental and theoretical methods at polyphenothiazine-modified $\mathrm{GC}^{[39]}$, and higher than the $k_{\mathrm{s}}=14 \mathrm{~s}^{-1}$ obtained at electrografted Azure-A modified graphene oxide ${ }^{[25]}$. Despite the limitations, the kinetic study performed at planar GC electrodes nevertheless provides insight into the superior electron transfer rate for electrografted vs. physically adsorbed Azure A groups.

The possibility to modulate and potentially achieve even higher loadings of electroactive groups and/or improved apparent electron transfer kinetics by electrografting was also explored. For these experiments, we varied either (i) the number of electrografting cycles or (ii) the switching potential. The characterisation $\mathrm{CV}$ secorded in $0.1 \mathrm{molL}^{-1}$ phosphate buffer $(\mathrm{pH}$ 7) solution at GC-CNT-AA Diaz electrodes are shown in Figure S5 and reveal the possibility to graft higher loadings of electroactive groups with increasing cycle number up to 100 cycles. Increasing the number of electrografting cycles from 1 to 10 increased the voltammetric peak areas without altering the sharp peak shape. For 50 and 100 electrografting cycles, a further increase in peak area is obtained together with the emergence of a second closely-overlapping peak. At 100 cycles, the maximum current density decreased slightly and the largest peak area is observed with the new overlapping peak becoming more evident. One explanation for these results is that the "thicker" polymeric assemblies (obtained with 50 or 
100 grafting cycles) exhibit mass transport and/or electron transfer limitations. The overlapping peaks may also correspond to the presence of two different but closely-related $\operatorname{active~centres~}^{[40]}$.

The use of different switching potentials revealed the possibility to obtain larger peak areas and therefore surface concentrations when scanning to moderate negative potentials $(-0.3 \mathrm{~V}$ and $-0.5 \mathrm{~V}$ vs. $\mathrm{Ag} / \mathrm{AgCl}$ ) compared to scanning to a negative potential of $-1.0 \mathrm{~V} v s$. $\mathrm{Ag} / \mathrm{AgCl}$. The latter switching potential of $-1.0 \mathrm{~V}$ resulting in a significant diminution of peak area and therefore apparent surface concentration. We attribute the lower surface concentration of Azure A groups to the occurrence, at least in part, of cross-reactions involving diazonium cations at potentials beyond $-0.5 \mathrm{~V} v s . \mathrm{Ag} / \mathrm{AgCl}$ that suppress film growth at the electrode surface. The irreversible reduction process observed at $-0.8 \mathrm{~V}$ vs. $\mathrm{Ag} / \mathrm{AgCl}$ may also be involved in film growth suppression or film degradation but was not investigated further (Figure S6). Breton, Gautier, and coworkers observed inhibited diazonium film growth at high overpotentials although this was in aprotic solvent ${ }^{[41]}$.

The bioelectrocatalytic glucose oxidation properties of the modified electrodes were subsequently investigated with immobilised fungal FAD-GDH. The use of diazonium-derived polyazine films for the electrical wiring of FAD-based enzymes for glucose oxidation has not been explored. GC-CNT-AA $A_{\text {Diaz }}$ and GC-CNT-AA $A_{\text {Ads }}$ bioelectrodes were prepared by incubation of the modified electrodes overnight in FAD-GDH solution then gentle rinsing to remove weakly-adsorbed enzyme (see Experimental Section). The bioelectrodes were characterised by cyclic voltammetry in $0.1 \mathrm{molL}^{-1}$ phosphate buffer ( $\mathrm{pH}$ 7) solution at a slow scan rate of $1 \mathrm{mV} \mathrm{s}^{-1}$ in the absence then presence of $100 \mathrm{mmolL}^{-1}$ glucose without stirring (Figure 3). At the GC-CNT-AA $A_{\text {Diaz }}$ bioelectrode, in the presence of glucose, an irreversible 
sigmoidal-like wave with an onset potential of $-0.05 \mathrm{~V} v s . \mathrm{Ag} / \mathrm{AgCl}$ is observed in the presence of glucose (Figure 3A). The bioelectrocatalytic wave is not observed in the absence of glucose, confirming effective bioelectrocatalytic oxidation of glucose linked to the polymer-type redox couple.
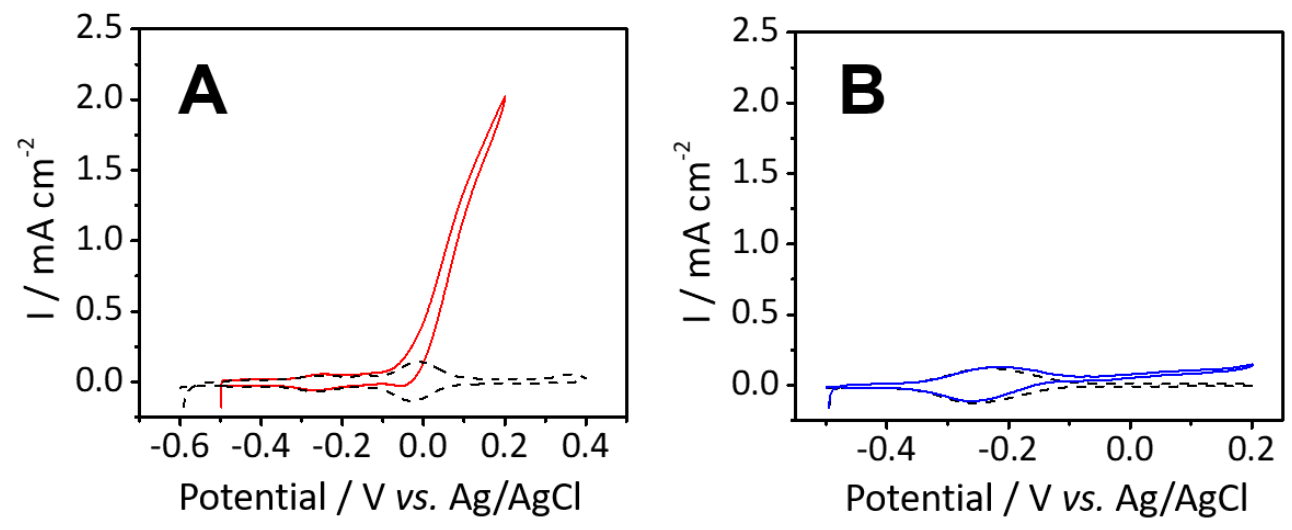

Figure 3. CVs recorded in $0.1 \mathrm{molL}^{-1}$ phosphate buffer (pH 7) after FAD-GDH immobilisation at (A) GC-CNT-AA Diaz bioelectrodes prepared by electrografting and (B) GCCNT-AA $A_{\text {Ads }}$ bioelectrodes prepared by physical adsorption, according to standard protocols. CVs recorded before (--) and after $(-,-)$ addition of $100 \mathrm{mmol}^{-1}$ glucose. Scan rate $=1 \mathrm{mV} \mathrm{s}$ 1

For the GC-CNT-AA Ads bioelectrode, in the presence of glucose, a small and ill-defined catalytic wave is observed with a similar onset potential to that observed for the electrografted electrode (Figure 3B). Maximum current densities of $2.0 \mathrm{~mA} \mathrm{~cm}{ }^{-2}$ and $0.15 \mathrm{~mA} \mathrm{~cm}^{-2}$ at $0.2 \mathrm{~V}$ vs. $\mathrm{Ag} / \mathrm{AgCl}$ were observed for the GC-CNT-AA $\mathrm{Diaz}_{\text {and }}$ GC-CNT-AA $\mathrm{A}_{\mathrm{Ads}}$ bioelectrodes, respectively, highlighting superior mediated bioelectrocatalysis for the electrografted Azure A bioelectrode. The data obtained in Figure 3 very clearly illustrates the importance of electrografted polymer-type rather than monomer-type and/or adsorbed azure A groups for electron mediation with FAD-GDH at CNT electrodes. The onset potentials are $c a$. 50-150 $\mathrm{mV}$ more positive compared to FAD-GDH bioelectrodes with thionine-polynorbornene ${ }^{[16]}$, 
phenanothroline quinone ${ }^{[6]}$ or 1,2 naphthoquinone hydroge ${ }^{[5]}$. However, the onset potential is still suitably low for glucose-oxidising bioanodes being similar to that observed with a 1,4 naphthoquinone hydrogel ${ }^{[13]}$ or $\mathrm{Ru}$ and Os complexes ${ }^{[7,11,12]}$. The observed onset potential at GC-CNT-AA $A_{\text {Diaz }}$ is also a major improvement compared to the $c a .0 .2 \mathrm{~V}$ vs. $\mathrm{Ag} / \mathrm{AgCl}$ observed at anodically grafted polyazine-MgOC electrodes with FAD-GDH ${ }^{[18]}$.

Amperometric measurements at $E_{\mathrm{p}}=0.2 \mathrm{~V}$ vs $\mathrm{Ag} / \mathrm{AgCl}$ (sat. $\mathrm{KCl}$ ) were subsequently performed at GC-CNT-AA $A_{\text {Diaz }}$ bioelectrodes to probe further the bioelectrocatalytic behaviour. The amperometric data obtained under hydrodynamic conditions in the presence of increasing glucose concentrations is shown in Figure 4. We note that such a current spike and slow decay to steady state is consistent with the injection of glucose which transiently induces a higher concentration in the vicinity of the electrode at the start of the experiment before homogenisation. A Michaelis Menten dependence with a $K_{M}$ value of $25 \mathrm{mmolL}^{-1}$ and limiting glucose concentration of $c a .75 \mathrm{mmol} \mathrm{L}^{-1}$ is observed. The enzyme-substrate kinetics are very similar with those observed for thionine-based FAD-GDH bioelectrodes ${ }^{[16]}$. This finding is consistent with previous work demonstrating that phenothiazines with little structural and potential difference did not show great affinity differences for FAD-GDH (Note: in this referenced study the enzyme and mediator was present in solution) ${ }^{[15]}$. Maximum current densities from repeat measurements were observed up to $2.0 \mathrm{~mA} \mathrm{~cm} \mathrm{cor}^{-2}$ for GC-CNT-AA $A_{\text {Diaz }}$ and hence improved mass transport via convection at $700 \mathrm{rpm}$ did not improve catalytic performance. 

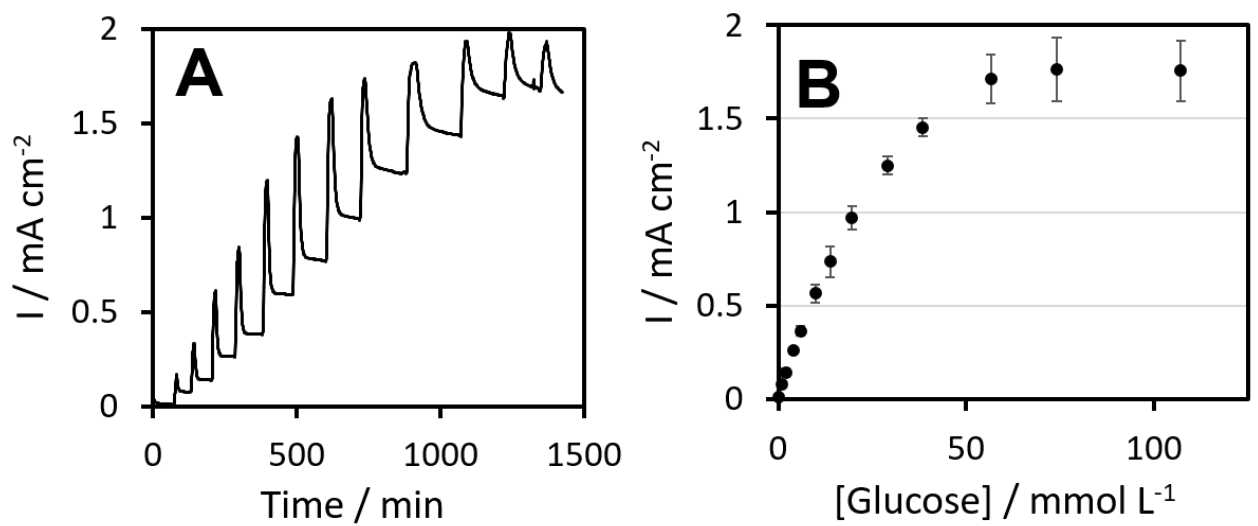

Figure 4. Amperometric data recorded in $0.1 \mathrm{~mol}^{-1}$ phosphate buffer $(\mathrm{pH} 7)$ at $E_{\mathrm{p}}=0.2 \mathrm{~V} v s$. $\mathrm{Ag} / \mathrm{AgCl}$ (sat. $\mathrm{KCl}$ ) at $\mathrm{GC}-\mathrm{CNT}-\mathrm{AA}$ Diaz bioelectrodes with immobilised FAD-GDH: (A) Amperogram and (B) corresponding plot showing current evolution with increasing glucose concentrations with stirring at $700 \mathrm{rpm}$. Error bars correspond to a $90 \%$ confidence interval from $n=3$ samples.

We also investigated the bioelectrocatalytic performance of GC-CNT-AA Diaz electrodes prepared by varying (i) the number of electrografting cycles and (ii) the switching potential. The maximum catalytic current was each time in the range of 1.6 to $2.0 \mathrm{~mA} \mathrm{~cm}^{-2}$ at $0.2 \mathrm{~V} v s$. $\mathrm{Ag} / \mathrm{AgCl}$ for the bioelectrodes prepared by varying the electrografting cycle number $(1,2,5$, $10,50,100$ cycles). No correlation between "thinner" (lower surface coverage) and "thicker" (higher surface coverage) films was observed. In contrast, the overpotential had a noticeable

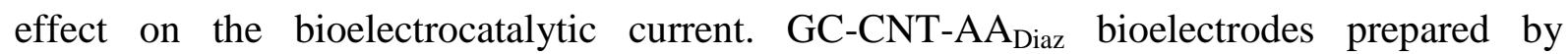
electrografting to a high overpotential of $-1.0 \mathrm{~V}$ only gave a catalytic current maximum of $c a$. $1.2 \mathrm{~mA} \mathrm{~cm} \mathrm{~cm}^{-2}$ compared to $c a .2 .0 \mathrm{~mA} \mathrm{~cm} \mathrm{~cm}^{-2}$ for the bioelectrodes prepared with an overpotential of $-0.5 \mathrm{~V}$ or $-0.3 \mathrm{~V} v s . \mathrm{Ag} / \mathrm{AgCl}$. The diminished catalytic performance reflects the, at least, 3-fold lower surface concentration at high overpotential (switching potential = 1.0 V vs. $\mathrm{Ag} / \mathrm{AgCl})$ to those prepared at lower overpotential.

Next we made experiments to explore the catalytic stability of GC-CNT-AA Diaz and GCCNT-AA $A_{\text {Ads }}$ electrodes in $0.1 \mathrm{~mol}^{-1}$ phosphate buffer $(\mathrm{pH} 7)$ in the presence of $100 \mathrm{mmol}^{-1}$ glucose. Amperometric measurements were recorded at $E_{\mathrm{p}}=0.2 \mathrm{~V} v s . \mathrm{Ag} / \mathrm{AgCl}$ for $15 \mathrm{~min}$ 
periods in freshly prepared solutions on different days during one week. Figure S7 shows typical amperometric data recorded at GC-CNT-AA $A_{\text {Diaz }}$ electrodes. The bioelectrodes were stored in fresh phosphate buffer $(\mathrm{pH} 7)$ at $4^{\circ} \mathrm{C}$ between experiments. The GC-CNT-AA Diaz electrodes exhibited only ca. $20 \%$ of the initial catalytic current after 2 days and $<10 \%$ after 5 days. We previously observed similarly low storage-type stabilities for the same enzyme over the period of one week. For example, $20-35 \%$ of the initial activity after 7 days for pyrene-NHS and phenanthroline-quinone FAD-GDH bioelectrodes prepared via covalent and non-covalent modification, respectively ${ }^{[6,16]}$. These results indicate that the low stability of the FAD-GDH bioelectrodes is related to the deactivation and/or leaching of the enzyme as opposed to the nature of the bonding between the enzyme and the electrodes and/or the mediator and the electrode (covalent vs. non-covalent). Furthermore, the potential electrostatic interaction between positively charged Azure A groups and negatively charged FAD-GDH at $\mathrm{pH} 7$ (isoelectric point 4.4) does not seem to contribute favourably to bioelectrode stabilisation.

\section{Conclusion}

Azure A groups can be readily electrografted or physically adsorbed at multiwalled carbon nanotube electrodes to give redox-active electrodes with similarly good stability and high surface coverage. The strategy of electrografting provided access to a polymer-type redox couple that could not be achieved using simple physical adsorption with a more attractive potential and electron transfer kinetics for electrically-wiring of the FAD-based enzyme. The formation of the higher potential polymer-type system was critical to achieve effective 
bioelectrocatalytic currents. The reported electrografting strategy however provided no obvious advantage in terms of bioelectrode stability compared to the electrode. Future work is now required to address the limited stability, for example, through the optimisation of the 3D porous electrode structure. Furthermore, the reported diazonium grafting approach could be investigated to introduce alternative "polymer-type" assemblies with improved bioelectrocatalytic driving force compared to the adsorbed "monomer-type" redox mediators that exist throughout the literature.

\section{Acknowledgments}

This work was supported by the ANR-JST program MOCCA-CELL (ANR-15-JTIC-000201) and grants from JSPS KAKENHI (18H01719). The authors also gratefully acknowledge the support from the platform Chimie NanoBio ICMG FR 2607 (PCN-ICMG), from the LabEx ARCANE (ANR-11-LABX-0003-01 and CBH-EUR-GS, ANR-17-EURE-0003), and from the Institut Carnot PolyNat (CARN 0007-01).

\section{References}

[1] A. J. Gross, M. Holzinger, S. Cosnier, Energy Environ. Sci. 2018, 11, 1670-1687.

[2] M. Holzinger, A. Le Goff, S. Cosnier, Electrochim. Acta 2012, 82, 179-190.

[3] P. Bollella, I. Lee, D. Blaauw, E. Katz, ChemPhysChem 2020, 21, 120-128.

[4] C. Abreu, Y. Nedellec, O. Ondel, F. Buret, S. Cosnier, A. Le Goff, M. Holzinger, Sens. Actuators B Chem. 2018, 277, 360-364.

[5] R. D. Milton, D. P. Hickey, S. Abdellaoui, K. Lim, F. Wu, B. Tan, S. D. Minteer, Chem. Sci. 2015, 6, 4867-4875.

[6] A. J. Gross, X. Chen, F. Giroud, C. Abreu, A. Le Goff, M. Holzinger, S. Cosnier, ACS Catal. 2017, 7, 4408-4416.

[7] M. N. Zafar, N. Beden, D. Leech, C. Sygmund, R. Ludwig, L. Gorton, Anal. Bioanal. Chem. 2012, 402, 2069-2077.

[8] R. D. Milton, F. Giroud, A. E. Thumser, S. D. Minteer, R. C. T. Slade, Phys. Chem. Chem. Phys. 2013, 15, 19371-19379.

[9] R. D. Milton, F. Giroud, A. E. Thumser, S. D. Minteer, R. C. T. Slade, Chem. Commun. 2014, 50, 94-96.

[10] A. Suzuki, N. Mano, S. Tsujimura, Electrochim. Acta 2017, 232, 581-585.

[11] L. Fritea, A. J. Gross, B. Reuillard, K. Gorgy, S. Cosnier, A. Le Goff, ChemElectroChem 2019, 6, 3621-3626. 
[12] R. Sakuta, K. Takeda, T. Ishida, K. Igarashi, M. Samejima, N. Nakamura, H. Ohno, Electrochem. Commun. 2015, 56, 75-78.

[13] C. Hou, Q. Lang, A. Liu, Electrochim. Acta 2016, 211, 663-670.

[14] P.-Y. Blanchard, P. H. M. Buzzetti, B. Davies, Y. Nedellec, E. M. Girotto, A. J. Gross, A. Le Goff, Y. Nishina, S. Cosnier, M. Holzinger, ChemElectroChem 2019, 6, 5242-5247.

[15] N. Tsuruoka, T. Sadakane, R. Hayashi, S. Tsujimura, Int. J. Mol. Sci. 2017, 18.

[16] L. Fritea, A. J. Gross, K. Gorgy, R. K. O’Reilly, A. Le Goff, S. Cosnier, J. Mater. Chem. A 2019, 7, 1447-1450.

[17] S. Pöller, M. Shao, C. Sygmund, R. Ludwig, W. Schuhmann, Electrochim. Acta 2013, $110,152-158$.

[18] N. Tsuruoka, S. S. Soto, A. B. Tahar, A. Zebda, S. Tsujimura, Colloids Surf. B Biointerfaces 2020, 192, 111065.

[19] T. Breton, A. J. Downard, Aust. J. Chem. 2017, 70, 960-972.

[20] A. J. Gross, V. Nock, M. I. J. Polson, M. M. Alkaisi, A. J. Downard, Angew. Chem. Int. Ed. 2013, 52, 10261-10264.

[21] M. M. Chehimi, Ed. , Aryl Diazonium Salts: New Coupling Agents and Surface Science, Wiley-VCH, Weinheim, 2012.

[22] T. Menanteau, M. Dias, E. Levillain, A. J. Downard, T. Breton, J. Phys. Chem. C 2016, 120, 4423-4429.

[23] A. Bensghaïer, F. Mousli, A. Lamouri, P. S. Postnikov, M. M. Chehimi, Chem. Afr. 2020, DOI 10.1007/s42250-020-00144-5.

[24] J. L. Bahr, J. P. Yang, D. V. Kosynkin, M. J. Bronikowski, R. E. Smalley, J. M. Tour, J. Am. Chem. Soc. 2001, 123, 6536-6542.

[25] C. Gómez-Anquela, M. Revenga-Parra, J. M. Abad, A. G. Marín, J. L. Pau, F. Pariente, J. Piqueras, E. Lorenzo, Electrochim. Acta 2014, 116, 59-68.

[26] G. Schmidt, S. Gallon, S. Esnouf, J.-P. Bourgoin, P. Chenevier, Chem. - A Eur. J. 2009, 15, 2101-2110.

[27] A. Bensghaïer, S. Lau Truong, M. Seydou, A. Lamouri, E. Leroy, M. Mičušik, K. Forro, M. Beji, J. Pinson, M. Omastová, M. M. Chehimi, Langmuir 2017, 33, 6677-6690.

[28] M. Revenga-Parra, C. Gómez-Anquela, T. García-Mendiola, E. Gonzalez, F. Pariente, E. Lorenzo, Anal. Chim. Acta 2012, 747, 84-91.

[29] N. Lalaoui, M. Holzinger, A. Le Goff, S. Cosnier, Chem. - A Eur. J. 2016, 22, 1049410500.

[30] R. Ortiz, R. Ludwig, L. Gorton, ChemElectroChem 2014, 1, 1948-1956.

[31] A. E. Blackwell, M. J. Moehlenbrock, J. R. Worsham, S. D. Minteer, J. Nanosci. Nanotechnol. 2009, 9, 1714-1721.

[32] A. Mesnage, X. Lefèvre, P. Jégou, G. Deniau, S. Palacin, Langmuir 2012, 28, $11767-$ 11778.

[33] D. Benito, C. Gabrielli, J. J. García-Jareño, M. Keddam, H. Perrot, F. Vicente, Electrochim. Acta 2003, 48, 4039-4048.

[34] A. A. Karyakin, in Electropolymerization (Eds.: S. Cosnier, A. Karyakin), WileyVCH Verlag GmbH \& Co. KGaA, Weinheim, Germany, 2010, pp. 93-110.

[35] E. Kalyoncu, M. Alanyalığlu, J. Electroanal. Chem. 2011, 660, 133-139.

[36] A. A. Karyakin, E. E. Karyakina, H.-L. Schmidt, Electroanalysis 1999, 11, 149-155. 
[37] K. Chiang Lin, A. T. E. Vilian, S. Ming Chen, RSC Adv. 2014, 4, 45566-45574.

[38] E. Laviron, J. Electroanal. Chem. Interfacial Electrochem. 1979, 101, 19-28.

[39] D. M. Gligor, M. L. Ungureşan, J. Math. Chem. 2010, 47, 1476-1482.

[40] J. Agrisuelas, C. Gabrielli, J. J. García-Jareño, D. Giménez-Romero, H. Perrot, F. Vicente, J. Phys. Chem. C 2007, 111, 14230-14237.

[41] L. Pichereau, I. López, M. Cesbron, S. Dabos-Seignon, C. Gautier, T. Breton, Chem. Commun. 2019, 55, 455-457.

[42] D. J. Garrett, P. Jenkins, M. I. J. Polson, D. Leech, K. H. R. Baronian, A. J. Downard, Electrochim. Acta 2011, 56, 2213-2220. 